\title{
A SYSTEM DIAGNOSIS THROUGH THE RECOGNITION OF THE MODE OF DYNAMIC MOTIONS OF A SHAFT BY THE INDIRECT MEASUREMENT IN JOURNAL BEARING
}

\author{
Rai-Wung Park* \\ Department of Technology Education, Mechatronics, Daebul University, Jeonnam, Korea \\ * Corresponding author (park1@db.ac.kr)
}

\begin{abstract}
This work presents a method to develop a system diagnosis regarding to shaft in journal bearing. The timely righteous estimation and judgment of the states is very important for the safe operation in time domain system. The synthesis of this problem presumes that all state variables are observable for concerned system. But it seems that it is not feasible to measure all modes of states or velocities, accelerations directly. A practical solution to this problem, which should be tried, is the estimation of the states or its velocities by the use of an observer which can estimate a system characteristics of linear or nonlinear states and effects as a mode, so called, “Indirect measurement". For this procedure, the mathematical model of the concerned physical system which consists of a long shaft and two journal bearings at the ends of the shaft, is derived with the significant remarks such as friction, gravitation, and Coriolis force. This is a basic system. The concrete assignment is to design the observer that estimates the characteristics of the states and velocities based on the measurement vectors. The main artifice is to approximate the characteristics with a fictitious model that may describe the modes of system errors. As a practical and convenient fictitious model, the characteristics of nonlinear effects are assumed as approximately stepwise contact. An identity observer is obtained whose state variables are the estimate of the state variable of the corresponding "observer system". It consists of a simulated model with a correction feedback of the estimation
\end{abstract}

Keywords: Diagnosis, Observer, Mode, Indirect Measurement, Journal, Bearing

\section{INTRODUCTION}

From the viewpoint of the increasing-complexity and high requirement of the structure of modern control systems, the reliability of a system is strongly considered. Especially, when the interest comes to the dynamic behavior by high signal amplitude or various operating points, the safe operation is getting more and more important. This is obviously verified when a part of systems suddenly goes out of function. It can cause an entire system defect, and this is able to bring up a dangerous situation for employee and material losses. To take precautions against these kinds of troubles, many scientists in the world have been making efforts for long time. For this, it is required the inspection during the operation: certainty and system assessment. Only by this way, the sudden appearance of defects and the alteration of the processes can be founded out, and the reason for the troubles and the place are detected(Fault Detection and Isolation, FDI). It is important that the fault must be sensed early enough for avoiding the damages in the system (Fault Detection, Isolation and Accommodation, FDIA). When this process goes without man's help, it's called automated fault detection, in the other word, "System Diagnosis". Under meaning of fault, we can understand every abnormal derivations or divergences from the required process behavior and the abrupt fault is meaningful of the safe operation. The FDI process needs certain characteristics being able to give the threshold of the decision : Residuum, residue. The method of fault estimate by using the Hardware-redundancy is very costly and less practicable. The model-based methods were presented by[1]. The methods by creating the parameters, or state space estimate 
were given in [2]. There have been some other ways to settle the problem with fault through the consideration of the property of the robustness [3]. A practical solution to this problem was the estimation of the states or its velocities by the use of observers which can estimate a system characteristics of linear or nonlinear states and effects as a mode, so called, "Indirect measurement"[4]. In this work, for this procedure, the mathematical model of the concerned physical system which in the operation time consists of a long shaft and two journal bearing at the ends of the shaft, is derived with the significant remarks such as friction, gravitation, and Coriolis force. With the indirect measurements on two bearing, an observer is designed and is going to use for the system diagnosis through the comparison with phases.

\section{Modeling of driver dynamic with constraind rotor and journal bearing}

In order to control and diagnose complex systems, one would like to obtain quantitative mathematical model of a system by symbolic representationinvolving an abstract mathematical formulation. Though a mathematical model can be adequate for a certain purpose in mind, it never describes the physical phenomenon exactly. Since the final goal of this work is to develop a diagnosis strategy, the inevitable conclusion is that the modeling problem and the diagnosis problem are not independent. Here, the necessary adequate models for the proposed diagnosis policies are built. These were comprised of the rotor model, the environment model, and model for the interaction between shaft and bearings. Industrial rotors are usually composed of shafts connected by couplings into a kinematic chain with the journal bearing in the operation situation.The shafts can be either cylindrical or revolute, and are driven by given actuators. In this work only cylindrical shaft will be considered. For the purpose of modeling, the three interacting parts such as the actuators, the transmission, and the bearing will be studied first separately and then, the complete model will be built.
The actuators are the devices that make the rotor move. There are some kinds of devices in the field, such as DC motors, pneumatic pistons, hydraulic piston, step motor, and $\mathrm{AC}$ server motors to mention the main possibilities. Since most of the present rotors employ AC server motors, detailed model for this actuator will be derived and used throughout this work. From now on, the index will be left out with respect to the whole dynamic system. The dynamic behavior of joint system can be modeled by analogy to [9] as follows.
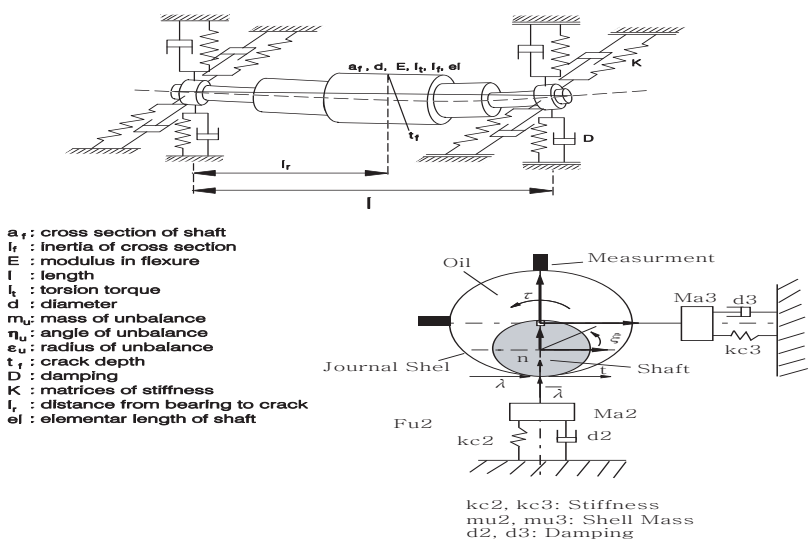

Fig. 1. A constraint system with a shaft and hydro-bearing

\subsection{Equation of motion}

$$
\begin{aligned}
& {\left[\begin{array}{cccc}
m & 0 & 0 & 0 \\
0 & m & 0 & 0 \\
0 & 0 & \theta & 0 \\
0 & 0 & 0 & \theta
\end{array}\right]\left[\begin{array}{c}
\ddot{U}_{x} \\
\ddot{U}_{y} \\
\ddot{U}_{c x} \\
\ddot{U}_{z y}
\end{array}\right]+\left[\begin{array}{cccc}
d & -G_{x y} & 0 & 0 \\
0 & d & 0 & 0 \\
-G_{x y} & 0 & d & -\Omega \theta_{p} \\
0 & 0 & -\Omega \theta_{p} & d
\end{array}\right]\left[\begin{array}{c}
\dot{U}_{x} \\
\dot{U}_{y} \\
\dot{U}_{c x} \\
\dot{U}_{z y}
\end{array}\right]+} \\
& {\left[\begin{array}{cccc}
K_{x x} & 0 & 0 & 0 \\
0 & K_{y y} & 0 & 0 \\
0 & 0 & K_{y x} & 0 \\
0 & 0 & 0 & K_{x y}
\end{array}\right]\left[\begin{array}{c}
U_{x} \\
U_{y} \\
U_{\varepsilon x} \\
U_{z y}
\end{array}\right]=m g\left[\begin{array}{l}
0 \\
1 \\
0 \\
0
\end{array}\right]+\left(\varepsilon_{u} \Omega^{2}\left[\begin{array}{c}
\cos \left(\Omega t+\eta_{u}\right) \\
\sin \left(\Omega t+\eta_{u}\right) \\
0 \\
0
\end{array}\right]\right.}
\end{aligned}
$$

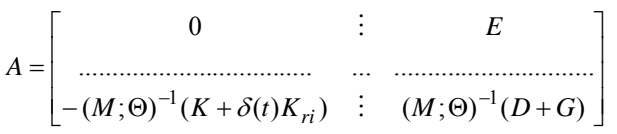

$$
\begin{aligned}
& f_{b}=\left[\begin{array}{c}
0 \\
\cdots \ldots \ldots \ldots \ldots \ldots \ldots \ldots \ldots \ldots \\
-(M ; \Theta)^{-1} B
\end{array}\right] \\
& y=\left[\begin{array}{cc}
C_{a} & 0 \\
0 & C_{g}
\end{array}\right] \\
& B=\left[\begin{array}{cccccc}
0 & 0 & 0 & f a k 1 & 0 & 0 \\
0 & 0 & f a k 2 & 0 & 0 & 0
\end{array}\right]^{T}(5)
\end{aligned}
$$


The factors fak1 and fak 2 denote mass unbalances in $\mathrm{x}$ and $\mathrm{y}$ coordinates respectively as follows

$$
f a k 1=\varepsilon m \Omega^{2} \cos \left(\Omega t+\eta_{u}\right)
$$

$f a k 2=\varepsilon m \Omega^{2} \sin \left(\Omega t+\eta_{u}\right)$

Die mass as an input is given as follows

$$
f g=\left[\begin{array}{llllllll}
0 & m g & 0 & 0 & 0 & m g & 0 & 0
\end{array}\right]^{T}
$$

It is normally convenient for further operation to write the equation above via state space notation with $x(t)=\left[\begin{array}{ll}U(t)^{T} & \dot{U}(t)^{T}\end{array}\right]$ including the nonlinearities of the motion created by any defects in system.

$\dot{x}(t)=A x(t)+u(t)+N_{R} n_{R}(x(t))+N_{u} n_{u}(t)$

The equation of the measurement is given by

$y=C x(t) \quad(10)$

where, $A$ is $\left(N_{n} \mathrm{X} N_{n}\right)$ dimensional system matrix which is responsible for the system dynamic with $N_{n}=2 n n, u(t)$ denotes $r$ dimensional vector of the excitation inputs due to gravitation and unbalances, and $C$ presents $\left(m_{e} \mathrm{X}_{N_{n}}\right)$ dimensional measurement matrix, respectively.

Here, the vector $n_{R}(x(t))$ and $n_{u}(x(t))$ characterizes the $n_{f}$ dimensional vector of nonlinear functions due to the fault such as mass unbalance and crack, respectively. $N_{u}$ and $N_{R}$ are the input matrices of the linear and the nonlinearities, and the order of ${ }_{N_{R}}$ is of $\left(N_{n} \mathrm{x} n_{f}\right)$. It is presupposed that the matrices $A, B, C, N_{R}$, the vector $u(t)$ and $y(t)$ are already known.

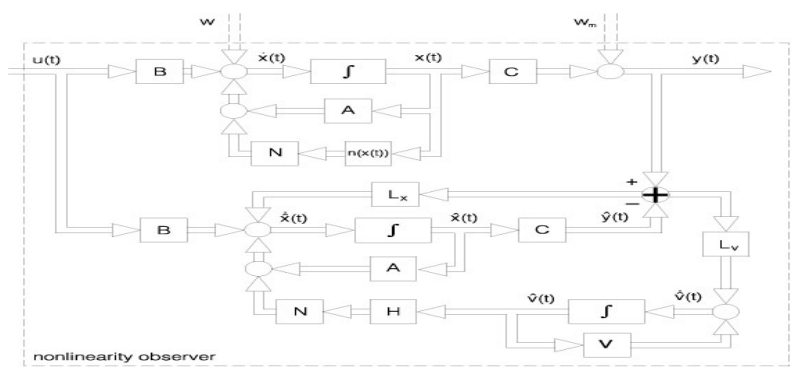

Fig.2. Nonlinear observer

Now it remains to reconstruct the unknown linear vector $n_{u}(x(t), t)$ and nonlinear vector $n_{R}(x(t), t)$ which mentions the disturbance force caused by a fault such as mass unbalance and crack. The basic idea is to get the signals from $n_{R}(x(t))$ approximated by the linear fictitious model, see [4, 5,
$6,7]$.

In terms of the fictitious model [4, 5], an observer [7] is designed and to get a signal phase an estimator is established.

$$
\begin{aligned}
{\left[\begin{array}{c}
\dot{\hat{x}}_{i}(t) \\
\dot{\hat{v}}_{1}(t) \\
\dot{\hat{v}}_{2}(t)
\end{array}\right]=} & {\left[\begin{array}{ccc}
A-L_{x} C & N_{R} H_{1} & N_{u} H_{2} \\
-L_{v 1} C & V_{1} & 0 \\
-L_{v 2} C & 0 & V_{2}
\end{array}\right]\left[\begin{array}{l}
\hat{x}_{i}(t) \\
\hat{v}_{1}(t) \\
\hat{v}_{2}(t)
\end{array}\right]+} \\
& {\left[\begin{array}{l}
I \\
0 \\
0
\end{array}\right] u(t)+\left[\begin{array}{c}
L_{x} \\
L_{v 1} \\
L_{v 2}
\end{array}\right] y(t) }
\end{aligned}
$$

For the guarantee of the observer ability of estimator, the requirement

$$
\operatorname{rank}\left[\begin{array}{ccc}
-\lambda I_{N_{n}}-A & -N_{v 1} H_{1} & -N_{u n} H_{2} \\
0 & \lambda I_{s 1}-V 1 & 0 \\
0 & 0 & \lambda I_{s 2}-V_{2} \\
C & 0 & 0
\end{array}\right]
$$

$$
\begin{aligned}
& =\operatorname{dim}(x(t))+\operatorname{dim}\left(v_{1}(t)\right)+\operatorname{dim}\left(v_{2}(t)\right) \\
& =N_{n}+n_{f}+2 n_{f} \quad \forall \lambda \in C^{+}
\end{aligned}
$$

and the requirement of the control ability

$$
\operatorname{rank}\left[\lambda I_{N_{n}}-A \quad B\right]=N_{n}
$$

must be satisfied. The output equation for the measurement is presented as follows.

$$
\hat{y}(t)=\left[\begin{array}{lll}
C & 0 & 0
\end{array}\right]\left[\begin{array}{c}
x(t) \\
v_{1}(t) \\
v_{2}(t)
\end{array}\right]
$$

where matrices $L_{x}$ and $L_{v}$ are the gain matrix of the observer.

The above Eq. (11) means that the observer consists of a simulated model with a correction feedback of the estimation error between real and simulated measurements. The matrix $A_{o}$ has $\left(N_{n}+n_{f} \times N_{n}+n_{f}\right)$ dimensions and represents the dynamic behavior of the elementary observer. The asymptotic stability of the elementary observer can guaranteed by a suitable design of the gain matrices $L_{x}$ and $L_{v}$ which are possible under the conditions of detect ability or observability of the extended system. To enable the successful estimation under the asymptotic stability, the eigenvalue of the considered observer $\left(A_{0}\right)$ must be settled on the left side of the eigenvalue of the given system $\left(A_{e}\right)$ to make the dynamic of the observer faster than the dynamic of the system. The fictitious model of the fault behaviors is able to be designed using integrator model $[7,8]$ based on the chosen crack model as follows. The observer gain matrices $L_{x}$ and $L_{L_{v}}$ can be calculated by pole assignment or by the Riccatiequation [4] as 
follows.

$A P+P A^{T}-P C^{T} R_{m}^{-1} C P+Q=0$

$L_{v}\left[\begin{array}{c}L_{x} \\ \ldots \\ L_{v}\end{array}\right]=P C^{T} R_{m}^{-1}$

The weighting matrix $Q$ and $R_{m}$ has to be suitably chosen by the trial and errors.

\section{Design of an estimator for the fault}

In the above section it has been studied how to design the elementary estimator for the detection at a given local position. It means that a certain place on the shaft is initially given as the position. The elementary estimator on the bearing has to survey not only the assigned local position but also any other place on the shaft and to give the signals whether a fault exists or not. As it has been known, it is possible to detect the fault assigned certain place along on the shaft. In the case a fault appears at any subsystem in running time, it must be detected as well. But in many cases, it has been shown that it is impossible or very difficult to estimate the position of the fault at all subsystem on the shaft with one estimator. Generally, it depends on the number of the subsystem and the number of estimator. For the estimation of a position of mass unbalance or crack, an estimator bank based on estimator is designed. The main idea is to reconstruct the related forces of a mass unbalance or crack from certain local position to the arranged elementary estimator. This is main task in this section. The structure of the estimator considered is in the work [7] presented. It consists of a few elementary estimator depends on the number of the subsystem is modeled. Every elementary estimator which is distinguished from the distribution vector $L s_{\left(i_{e}\right)}$ gets the same input (excitation) $u(t)$ and the feedback of the measurements, and is going to be set up at a suitable place on the given system. For the appreciate arrangement of Beo, the distribution matrix on the analogy of (14) has been applied. In this way the estimator bank is established with the estimator. To estimate the local place of the fault, there are two steps. First of all, the estimator must be observable to certain local place in the meaning of the asymptotical stability in the system. The requirement has been satisfied by the criteria from $[4,7]$.

This means that the estimator has to be capable of estimating the fault at any location, where estimator is situated on the given system. The unknown fault position is to be found by the estimator arranged in a certain local place with the related crack forces resulting from the crack. To guarantee this condition (12) is supposed to be fulfilled. In this work three estimators are arranged on the left and right bearings

$$
L s(i)=\left[\begin{array}{lllll}
11 & , \cdots, & 0000 & , \cdots, & 11
\end{array}\right]^{T}
$$

The unknown position of a fault is found by the estimator according to the related forces, displacement, and torque of some other location on the shaft.

$$
\begin{aligned}
& {\left[\begin{array}{c}
\dot{\hat{x}}_{i}(t) \\
\hat{\hat{v}}_{1_{i}}(t) \\
\hat{\hat{v}}_{2_{i}}(t)
\end{array}\right]=\left[\begin{array}{ccc}
A-L_{x_{i}} C & N_{R i} H_{1} & N_{u_{i}} H_{2} \\
-L_{v 1 i} C & V_{1} & 0 \\
-L_{v 2_{i}} C & 0 & V_{2}
\end{array}\right]\left[\begin{array}{c}
\hat{x}_{i}(t) \\
\hat{v}_{l_{i}}(t) \\
\hat{v}_{2_{i}}(t)
\end{array}\right]+} \\
& {\left[\begin{array}{c}
I \\
0 \\
0
\end{array}\right] u(t)+\left[\begin{array}{c}
L_{x_{i}} \\
L_{v 1} \\
L_{v 2 i}
\end{array}\right] y(t), \quad i=2,4,6}
\end{aligned}
$$

\section{Simulation on hydrostatic rotating shaft}

The estimator bank consists of two elementary estimators. The $1^{\text {st }}$ estimator $\mathrm{A}$ is situated at the left bearing and $2^{\text {nd }}$ estimator $\mathrm{B}$ is placed at the right bearing. The criteria to detect a fault, it is necessary to choose the maximal magnitude of the phase from all estimator by the comparison among the phase turned out : forces, displacements and torque. In the case, the estimator shows none of the force, there is not any mass unbalance in this system considered. If any one of the estimator gives the signal of a force the system has a crack in a corresponding position. The figure illustrates the phases with the mass unbalance under the rpm (107.5). As the $1^{\text {st }}$ example, the nominal system behavior is considered with given mass unbalance is at the $1^{\text {st }}$ of the node in the system. 


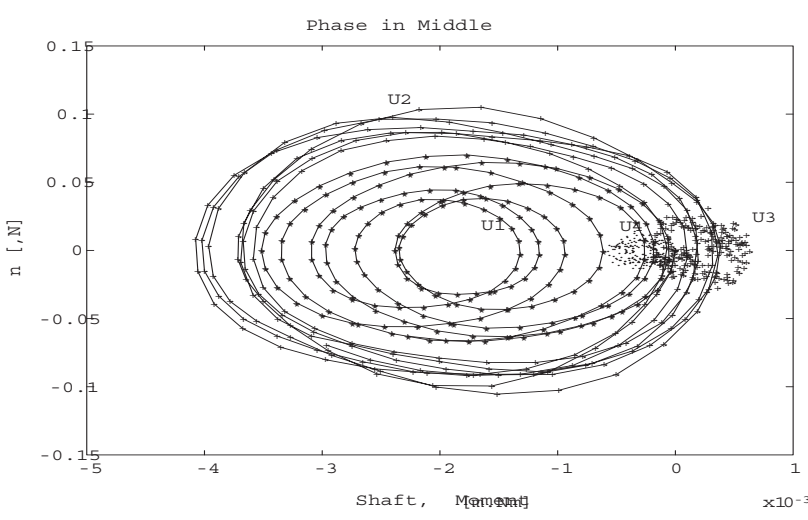

Fig. 3 Phase

The y axis shows the force which denotes nonlinearity and the $\mathrm{x}$ axis illustrates the corresponding torque. This phase is nominated as a phase of zero fault. The fig. 3 shows that the estimator recognizes the non existence of a fault. By the comparison of the forces, there is some difference between estimated and simulated phase. However, the derivation is small enough and acceptable.

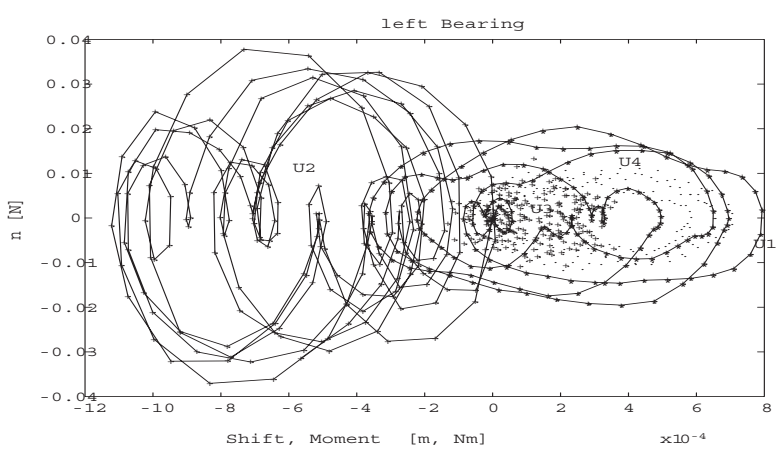

Fig. 4.Phase 2.

The results in the Fig. 4 describe the fault existence (force of mass unbalance) in the $2^{\text {nd }}$ node under the influence of a crack in the runtime operation. Up to $3[\mathrm{sec}]$, the forces of mass unbalance and crack have been overlapped. This denotes that mass unbalance and crack exist in the same place (node) on the shaft. It has been already mentioned that the breathing direction of a crack and the position of the mass unbalance on the radius of the diameter of shaft are on the same line. By the comparison the Fig. 3 with Fig. 4, it is clear to appear a defect.

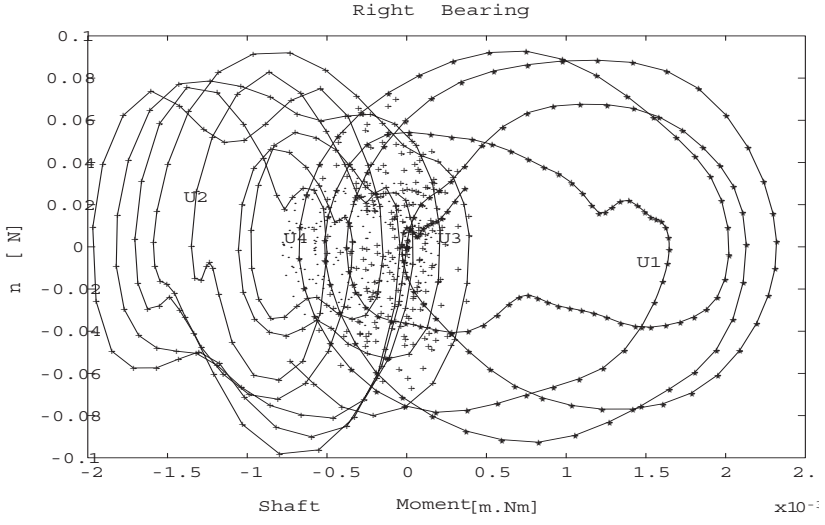

Fig. 5.Phase 3.

The result in the Fig. 5 illustrates the appearance of the fault as phase 4 th node: the forces of mass unbalance, in the middle of the shaft.

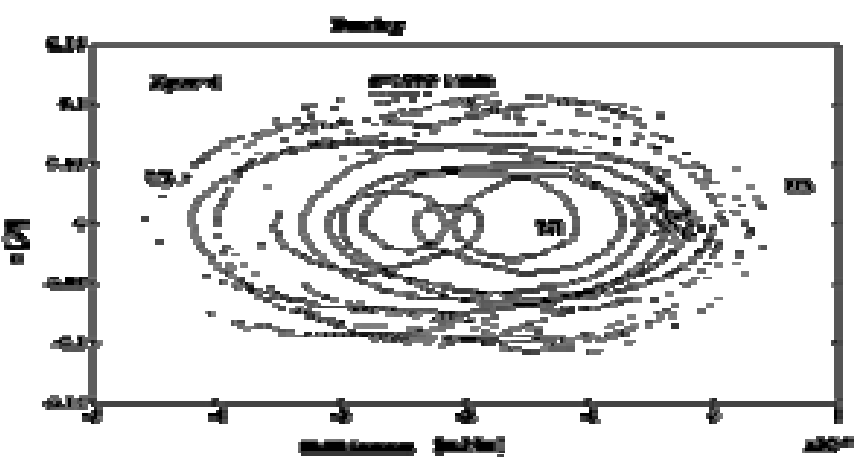

Fig. 6. Phase 4

The result in the Fig. 6 tells us the coming up of the fault as phase: the forces of mass unbalance, the forces of mass unbalance, in the $1^{\text {st }}$ node under the influence according to the same direction between mass unbalance and the crack on

\section{Conclusion}

From physical model, the mathematical model of the rotating shaft with bearings has been presented. Based on the mathematical model, the elementary observer with the measurement only on the bearingsand the observer bank have been developed. With this observer bank, the estimation of the fault has been detected in phase. The above method gives a clear relation between the shaft with a mass unbalance and the damaged shaft by a crack and the caused phenomena in phase by means of the measurement at both bearings. Successful theoretical results have been given in graphics. The forces and torques in the results are the internal one, which have been 
reconstructed as disturbance forces created by the mass unbalance and crack. It has been theoretically shown, that it is possible to estimate localization of a mass unbalance with the opposite direction of a crack. The suggested methods are very significant not only for the further theoretical research and development but also for thetransfer in experiments.

\section{References}

[1] R.V. Beard, Failure acommodation in linear system through self recognition, Rept. MVT-71-1, Man vehicle laboratory, M.I.T., Cambridge, Mass., Feburar(2005)

[2] R. J. Patton and S. M. Kangetehe, Robust faultdiagnosisusing eigenstructure assignment of observers, In R, Patton, $\mathrm{P}$.

[3] Frank, and R. Clark, Herausgeber, Fault diagnosis in dynamic systems, Prentice Hall (1984)

[4] R. Iserman, Identification dynamischersysteme, Band I and II, Spring Verlag, Berlin(1984)

[5] P.C. Mueller, Estimation and compensation of nonlinearities, Proc. Of $1^{\text {st }}$ Asian Confer.,Tokyo, (1994) 228-234.

[6] R.W. Park, Estimation of a mass unbalance under the crack on the rotating shaft, ICASE, Vol. 2, No. 4, (2000) 228-234.

[7] R.W. Park, Crack detection, localization and estimation of a depth in a turbo rotor, KSME International Journal, V01.14, No. 7, (2000) 732-729.

[7] R.W. Park and P.C. Mueller, A contribution to crack detection, localization and estimation of a depth in a turbo rotor, ASCC, proc. Vol. II, (1997) 427-430.

[8] R. W. Park and S. Cho, Noise and fault diagnosis using control theory, international session paper, Proc $13^{\text {th }}$ Korea Automation Control Conference, ICASE, (1998) 301-307.

[9] Bathe K. J. , Finite Elemente Method, Springer Verlag, 1990.

\section{Appendices}

The data used in the simulation are given as follows:

$\mathrm{A}_{(\mathrm{i}=1, \ldots 8: \mathrm{j}=1, \ldots 8)}=\left[\begin{array}{cccccccc}0 & 1 & 0 & 0 & 0 & 0 & 0 & 0 \\ 0 & n 1 & n 2 & n 3 & 0 & 0 & 0 & 0 \\ 0 & 1 & 0 & 0 & 0 & 0 & 0 & 0 \\ 0 & 1 & 0 & 0 & 0 & 0 & 0 & 0 \\ 0 & 1 & 0 & 0 & 0 & 0 & 0 & 0 \\ 0 & 1 & 0 & 0 & 0 & 0 & 0 & 0 \\ 0 & 1 & 0 & 0 & n 5 & n 6 & 0 & 1 \\ 0 & 1 & 0 & 0 & 0 & 0 & n 7 & n 8\end{array}\right]$

$A_{(i, j)}$ is the system matrix of order( $\left.64 \times 64\right)$ The length of subsystem of load mass makes el $=0.2 \mathrm{~m}$, diameter of the subsystem of the load mass $\mathrm{m}$ makes ed $=0.25 \mathrm{~m}$. The mass of load is of $\mathrm{m}=\pi \mathrm{el} \rho \mathrm{ed}^{2} / 4$, and the density makes $\rho=7860 \mathrm{~kg} / \mathrm{m}^{3}$. The nominated elements $n_{1}, n_{2}, n_{3}$, and $n_{4}$ are of $-0.0084,-0.8321,-0.3747,-0.0321$ respectively. The remained elementsn5, n6, $\mathrm{n} 7$ and $\mathrm{n} 8$ have the value of $-0.025,-0.754,-0.852$ and -0.459 . The matrices $\mathrm{M}, \mathrm{G}$, and d present the mass-, stiffness-, gravity- and damping matrix $[7,9]$. The others are of :fg $=f(g$, in $=1, \ldots, N)$ $+\mathrm{fu}=\mathrm{f}(\mathrm{g}$, in $=1, \ldots, 30,31,32)=0 ; \mathrm{f}(\mathrm{g}, 2$, '”, 29) $=-\mathrm{mg}$ $\mathrm{f}(\mathrm{u}, ;, 21)=\mathrm{f}(\mathrm{u}, ;, 25)=-\mathrm{em} \Omega \mathrm{m}(\mathrm{ex}) \sin \left(\Omega^{2} \mathrm{t}+\right.$ $\beta), \mathrm{f}(\mathrm{u}, ;, 18)=\mathrm{f}(\mathrm{u}, ;, 22)=\mathrm{em} \Omega \mathrm{m}(\mathrm{ex}) \cos \left(\Omega^{2} \mathrm{t}+\right.$ $\beta$ ), where the order of the fg and fu are of $(32 \times 1)$ respectively. The excentricity: $\mathrm{em}=0.001$, massof excentricity $\mathrm{e}(\mathrm{ex})=4 \mathrm{~mm}$. The modulus $\mathrm{E}$ is of $2.0 \mathrm{x}$ $10 \mathrm{~N} / \mathrm{mm}^{2}$. The measurement matrix $\mathrm{C}$ has the oder $(4 \mathrm{x} 66)$ $C_{a}=C_{g}=1$, otherwise $C(i, j)=0, \Theta=\Theta_{p}=0^{0}, \Omega=1 \mathrm{rad} / \mathrm{sec}$, $\varepsilon_{\mathrm{u}}=0.04, \dot{\eta}=0.71, \beta=0$

$\tau: 1.8, \mathrm{u}(\mathrm{t})=120 / \mathrm{mm} \quad \sin (\pi \mathrm{t} / \mathrm{s})[0:-1 / \mathrm{M}]_{(36 \mathrm{x} 1)}^{-1}$

The weighting matrix $Q$ is of $: Q(i, j)=3.10^{6}, i=j=1, \ldots$, $12, Q(i, j)=6.10^{5}, i=j=13, \ldots, 18, Q(i, j)=2.5 .10^{6}, \quad i=$ $j=19, \ldots, 28, Q(i, j)=2.9^{104}, \quad i=j=29, \ldots, 42, Q(i, j)=$ $3.10^{5}, i=j=43, \ldots, 63, Q(i, j)=4.10^{8}, \quad i=j=64, \ldots, 66$, 